\title{
Expression changes of Akt and GSK-3 $\beta$ during vascular inflammatory response and oxidative stress induced by high-fat diet in rats
}

\author{
Jingshang Wang ${ }^{1}$, Huijun Yin ${ }^{1 *}$, Ye Huang ${ }^{2}$, Chunyu Guo ${ }^{1}$, Chengdong Xia ${ }^{3}$, Lu Zhang ${ }^{1}$ \\ ${ }^{1}$ Department of Cardiovascular Disease, Xiyuan Hospital, China Academy of Chinese Medical Sciences, Beijing, China \\ ${ }^{2}$ Department of Emergency, Xiyuan Hospital, China Academy of Chinese Medical Sciences, Beijing, China \\ ${ }^{3}$ Department of Endocrinology, Xiyuan Hospital, China Academy of Chinese Medical Sciences, Beijing, China \\ Email: wangjingshang108325@126.com, ${ }^{*}$ huijunyin@yahoo.com.cn
}

Received 25 February 2013; revised 2 April 2013; accepted 4 May 2013

Copyright (C) 2013 Jingshang Wang et al. This is an open access article distributed under the Creative Commons Attribution License, which permits unrestricted use, distribution, and reproduction in any medium, provided the original work is properly cited.

\begin{abstract}
Aim: To observe the expression changes of Akt and GSK-3 $\beta$ during vascular inflammatory response and oxidative stress induced by high-fat diet in rats. Methods: 20 male Sprague-Dawley rats were separately fed for 18 weeks with two types of diets; a normal diet (control group, CON) or high-fat diet hyperlipidmia group, HLP). Then the body weight, lipid parameter, plasma hepatocyte growth factor (HGF), serum superoxide dismutase (SOD), malondialdehyde (MDA), Tumor necrosis factor- $\alpha$ (TNF- $\alpha$ ), a Soluble intercellular adhesion molecule-1 (sICAM-1), Lectin-like oxidized cellulose low density lipoprotein receptor-1 (LOX-1), as well as aortic endothelial p-GSK-3 $\beta$, GSK-3 $\beta$, p-Akt, Akt expressions were determined. Results: In comparison with the control group, the model group showed a significant increase in the levels of serum total cholesterol (TC), triglyceride (TG), low density lipoprotein cholesterol ( LDL-C) and significant decrease in the level of serum high density lipoprotein cholesterol (HDL-C) after high-fat diet for 18 weeks $(p<0.05$ or $p<0.01)$. Meanwhile, a more obvious increase of plasma HGF, LOX-1 and serum MDA, TNF- $\alpha$, and SICAM-1 levels were observed relative to the control group $(p<0.05$ or $p<$ 0.01). Moreover, high-fat diet significantly increased the phosphorylation of Akt and GSK-3 $\beta$ in rat aorta. Conclusion: Short-term high-fat diet could induce vascular endothelium injury by increasing inflammation and oxidative stress. And PI3K/Akt pathway could play an important role in hyperlipidemia-induced vascular endothelium injury.
\end{abstract}

"Corresponding author.
Keywords: High-Fat Diet; Akt and GSK-3 $\beta$; Vascular Inflammatory Response; Oxidative Stress; Endothelium Injury

\section{INTRODUCTION}

Atherosclerosis is the pathological basis of cardiovascular diseases which represent the leading cause of death worldwide; and recent epidemiological data have strongly suggested that hyperlipidemia, characterized by elevated serum total cholesterol and low density and very low density lipoprotein cholesterol and decreased high density lipoprotein, is an important risk factor for coronary heart disease and this increased risk appears to be independent of other known risk factors [1,2]. Recent research has shown that inflammation plays a key role in coronary artery disease and atherosclerosis development $[3,4]$. It also has become clear that oxidative stress represents a common pathogenic mechanism for atherosclerosis, from lipid streaks formation to plaque rupture and thrombosis. A particularly important mechanism for ROS-mediated atherosclerosis appears to be through stimulation of proinflammatory events [5]. Among all confirmed mechanisms, vascular endothelium injury is claimed to an important initial event at the onset of atherosclerosis [6].

Phosphatidylinositol-3-kinase (PI3K) and Akt are downstream effectors of insulin signaling as well as important signaling molecules in the regulation of glycogen metabolism in myocytes, lipocytes, and hepatocyte. By regulating angiogenesis, proliferation, microvascular permeability, survival, cellular transformation, and embryonic differentiation, PI3K/Akt also plays an important role in the regulation of endothelial cell functions [7]. Among the various intracellular downstream effectors of Akt, GSK- $3 \beta$ phosphorylation and inactivation are con- 
sidered important mechanisms of cell survival [8]. However, As yet, little is known about the role of Akt and GSK-3 $\beta$ expressions in the vascular endothelium inflammation and oxidative stress induced by hyperlipidemia.

Therefore, in the current study, we aimed to determine the influence of high-fat diet feeding on endothelial inflammation and oxidative stress in rats and thus to investigate the possible role of PI3K/Akt/GSK-3 $\beta$ pathway involved.

\section{MATERIALS AND METHODS}

\subsection{Drugs and Reagents}

Superoxide dismutase (SOD) and malondialdehyde (MDA) assay kits were obtained from Nanjing Jiancheng Bioengineering Institute, China. Enzyme-linked immunosorbent assay (ELISA) kits of soluble intercellular adhesion molecule 1 (sICAM-1), tumor necrosis factor $\alpha$ (TNF- $\alpha$ ), hepatocytegrowth factor (HGF) and Lectin-like oxidized cellulose low density lipoprotein receptor-1 (LOX-1) were all purchased from R\&D (USA). The antibodies against Akt, phosphorylated-Akt (Ser473), GSK$3 \beta$, phosphorylated-GSK $3 \beta$ (Ser9) and $\beta$-actin were purchased from Cell Signaling Technology (USA). All other biochemicals used were of the highest purity available.

\subsection{Establishment of Hyperlipidmia Model and Grouping}

20 male Sprague-Dawley rats, 180 - 220 g, were obtained from Vital River Laboratory Animal Technology Co. Ltd. Beijing. Rats were housed in the facility at Xiyuan hospital, China Academy of Chinese Medical Sciences according to the guidelines for laboratory animals approved by Beijing Experimental Animal Management Center. After 1 week of adaption, 10 rats were selected randomly to switch from normal diet (control group, $\mathrm{CON}$ ) to highfat diet (hyperlipidmia group, HLP) which contained $10 \%$ fat $(\mathrm{wt} / \mathrm{wt}), 10 \%$ sugar $(\mathrm{wt} / \mathrm{wt}), 2.5 \%$ cholesterol $(\mathrm{wt} / \mathrm{wt})$ and cholate $0.25 \%(\mathrm{wt} / \mathrm{wt})$ for 18 weeks.

The establishment of the hyperlipidmic rats was evaluated by analyzing blood lipid levels including TC, TG, LDL-C and HDL-C.

\subsection{Specimen Collection}

At the 20th week, all rats were executed after anesthesia with intraperitoneal injection of $20 \%$ urethane $(0.6 \mathrm{~mL} /$ $100 \mathrm{~g}$, fasting $12 \mathrm{~h}$ before sampling). Blood samples were collected from abdominal aorta. The aorta was removed for Westen-blot analysis.

\subsection{Assay for Lipid Parameter, MDA and SOD}

TC and TG were determined by enzymic technique.
HDL-C and LDL-C were analyzed by immunoturbidimetry. The activities of SOD and the concentration of MDA were both determined by using commercially available kits.

\subsection{ELISA Assay}

Serum TNF- $\alpha$, soluble ICAM-1 (sICAM-1) levels and plasma HGF and LOX-1 from rats were measured by ELISA according to the instructions from R\&D Systems (USA).

\subsection{Western Blot Analysis}

Total protein $(50 \mathrm{mg} / \mathrm{lane})$ from aorta was separated by SDS-PAGE and transferred to a polyvinylidene fluoride membrane. After incubation in blocking solution (5\% nonfat milk) (Sigma), membranes were incubated with primary antibodies for Akt, phosphorylated Akt, GSK$3 \beta$, phosphorylated-GSK- $3 \beta$ or $\beta$-actin for overnight at $4^{\circ} \mathrm{C}$. Membranes were washed and then incubated with 1:2000 dilution horseradish peroxidase-conjugated secondary antibody (ZSGB-BIO, Beijing, China). The relative density of each protein band was normalized to that of $\beta$-actin. All results were representative of at least 3 independent experiments.

\subsection{Statistical Analysis}

The results were presented as means \pm S.D. The SPSS statistics 15.0 package was utilized to analyze the data. Differences between these two groups were analyzed using $\mathrm{t}$ test. The $\mathrm{p}<0.05$ was considered statistically significant.

\section{RESULTS}

\subsection{Effect of High-Fat Diet on Body Weight and Serum Lipid Parameters}

As shown in Table 1, compared to the control, the body weight and the serum levels of TC, TG, LDL-C increased after high-fat diet for 18 weeks $(p<0.05$ or $p<$ $0.01)$, whereas the HDL-C level in sera decreased significantly $(\mathrm{p}<0.01)$.

\subsection{Effect of High-Fat Diet on Plasma HGF and LOX-1 Levels}

As shown in Figure 1, plasma HGF and LOX-1 levels showed a significant increase in rats with high-fat diet compared with the control $(\mathrm{p}<0.01)$.

\subsection{Effect of High-Fat Diet on Serum SOD and MDA Levels}

As shown in Table 2, after high-fat diet for 18 weeks, 
Table 1. Changes of body weight and serum lipid parameters.

\begin{tabular}{cccccc}
\hline Group & Body weight $(\mathrm{g})$ & TC $(\mathrm{mmol} / \mathrm{L})$ & TG $(\mathrm{mmol} / \mathrm{L})$ & HDL-C $(\mathrm{mmol} / \mathrm{L})$ & LDL-C $(\mathrm{mmol} / \mathrm{L})$ \\
\hline NOR & $577.83 \pm 40.10$ & $1.10 \pm 0.23$ & $0.66 \pm 0.23$ & $0.48 \pm 0.05$ & $0.34 \pm 0.07$ \\
HLP & $646.00 \pm 52.78^{*}$ & $3.78 \pm 0.16^{* *}$ & $1.58 \pm 0.16^{* *}$ & $0.28 \pm 0.06^{* *}$ & $0.63 \pm 0.09^{* *}$ \\
\hline
\end{tabular}

Data are presented as the means \pm S.D. $(\mathrm{n}=10) .{ }^{*} \mathrm{p}<0.05,{ }^{* *} \mathrm{p}<0.01$, compared with the control group.

Table 2. Effect of high-fat diet on serum SOD and MDA levels.

\begin{tabular}{ccc}
\hline Group & SOD (U/mg protein) & MDA (nmmol/mg protein) \\
\hline NOR & $43.79 \pm 11.48$ & $2.93 \pm 0.70$ \\
HLP & $27.50 \pm 2.52^{* *}$ & $5.15 \pm 0.63^{* *}$ \\
\hline
\end{tabular}

Data are presented as the means \pm S.D. $(n=10) .{ }^{* *} \mathrm{p}<0.01$, compared with the control group.

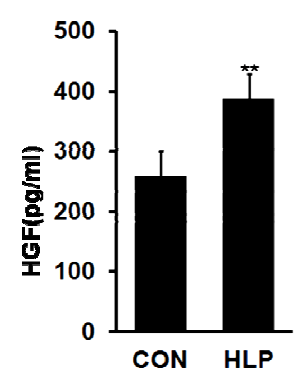

(a)

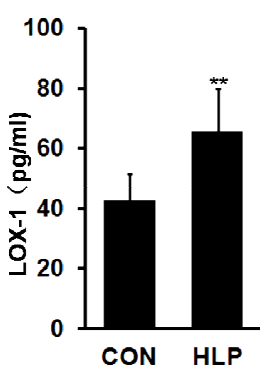

(b)
Figure 1. Changes in plasma hepatocyte growth factor (HGF, pg/ml) (a) and lectin-like oxidized cellulose low density lipoprotein receptor-1 (LOX-1, pg/ml) (b) levels after highfat diet for 18 weeks. Data are presented as the means \pm S.D. $(\mathrm{n}=10) .{ }^{* *} \mathrm{p}<0.01$, compared with the control group.

the serum level of SOD decreased significantly $(\mathrm{p}<$ 0.01 ), whereas MDA level increased obviously compared with the control $(\mathrm{p}<0.01)$.

\subsection{Effect of High-Fat Diet on Serum TNF- $\alpha$ and sICAM-1 Levels}

As shown in Figure 2, compared with the control, serum levels of TNF- $\alpha$ and sICAM-1 increased significantly (p $<0.01$ ), which indicated that inflammation activated and increased cell adhesion ability in rats with high-fat diet for 18 weeks.

\subsection{Effect of High-Fat Diet on Akt and GSK-3 $\beta$ Phosphorylation Levels}

To investigate the underlining mechanism of high fat diet induced endothelial injury accompanied by obvious inflammation and oxidative stress, we examined the expression changes of Akt and GSK- $3 \beta$ in rat aorta. As shown in Figure 3, in comparison with the control, the

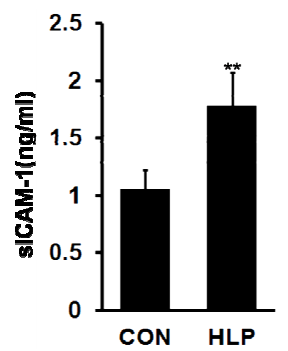

(a)

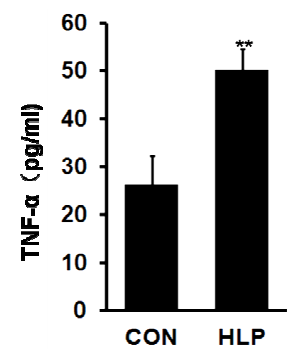

(b)
Figure 2. Changes in serum soluble intercellular adhesion molecule 1 (sICAM-1, ng/ml) (a) and tumor necrosis factor $\alpha$ (TNF- $\alpha, \mathrm{pg} / \mathrm{ml}$ ) (b) levels after high-fat diet for 18 weeks. Data are presented as the means \pm S.D. $(\mathrm{n}=10) .{ }^{* *} \mathrm{p}<0.01$, compared with the control group.

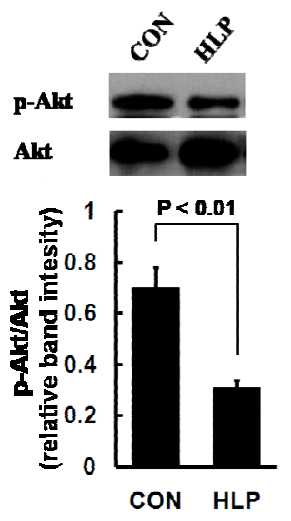

(a)

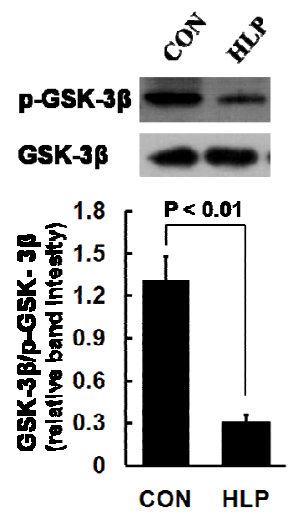

(b)
Figure 3. Phosphorylation of Akt (a) and GSK$3 \beta$ (b) in rat aorta was determined by Western blot. Data obtained from quantitative densitometry were presented as mean \pm S.D. of 3 independent experiments.

protein phosphorylation of Akt and GSK- $3 \beta$ decreased obviously in rats with high-fat diet.

\section{DISCUSSION}

Hyperlipidemia is an independent risk factor of atherosclerosis [1]. Epidemiological investigations so far have shown that the plasma cholesterol levels are increased in most patients with atherosclerosis and the severity of atherosclerosis is positively correlated with the increase of plasma low density lipoprotein and total cholesterol levels [2]. Animals fed a fatty diet with long term 
showed marked atherosclerosis $[9,10]$, further proof showed that hyperlipidemia played an important role in the atherosclerosis development. In recent years, it has become apparent that atherosclerosis is a chronic inflammatory process affecting large- and medium-sized arteries throughout the cardiovascular system. And inflammation has been recognized as an important initial event at the onset of atherosclerosis, meanwhile involved in the development of atherosclerosis [11]. Besides, some experts have pointed out that abnormal lipid metabolism and inflammation are the most promising targets for the atherosclerosis intervention. Therefore, it will give us more effective means for atherosclerosis intervention by exploring the explicit pro-inflammatory effects of hyperlipidemia and its detailed mechanism.

In our study, animal model of hyperlipidemia was verified by measurement of body weight and blood lipid levels. After high-fat diet for 18 weeks, we observed the obvious increase in body weight, TC, TG, and LDL-c levels and decreased HDL-c level in SD rats. Plasma HGF, which increases significantly along with the aggravation of injury, has been identified as a protective factor of vascular endothelial injury. So, herein HGF was used to evaluate the extent of vascular injury [12]. Our study showed that the plasma HGF level was increased obviously in rats with high-fat diet compared with the control, which indicated that a short-term high-fat diet can lead to obvious vascular endothelial injury.

Oxidative stress and inflammation are the main mechanisms of vascular endothelial injury, which has been thought as the initial step of atherosclerosis [13]. As known, MDA is a by-product of lipid peroxidation induced by excessive ROS and widely used as a biomarker of oxidative stress [14]. On the other hand, SOD, as an endogenous antioxidant, plays a pivotal role in preventing cellular damage caused by ROS and indicates antioxidant capacity of the body $[14,15]$. In our present study, marked MDA content increase and SOD activity decrease were found in rats with high-fat diet, which indicated that 18 weeks-high-fat diet can induce obvious oxidative stress in rats accompanied by significant antioxidant capacity decrease.

Previous experiments have shown that the elevated blood lipids can increase the phosphoinositide hydrolysis, synthesis and release of endothelin in endothelial cells, then activate the endothelial adhesion molecules secretion and promote the circulating monocytes adhesion to vascular well. Tumor necrosis factor $\alpha(\mathrm{TNF}-\alpha)$, as an important medium in inflammation and multiple pathophysiological processes, can lead to a series of inflammatory damage and mediate the adhesion of leukocytes to vascular endothelial cells [16]. Intercellular adhesion molecules (ICAMs), as one part of the immunoglobulin superfamily, play important role in inflammation, im- mune responses and in intracellular signalling events [17]. Animal experiments confirmed that the degree of atherosclerosis decreased significantly in Apolipoprotein E-deficient $\left(\mathrm{ApoE}^{--}\right)$mice with ICAM-1 knockout [18]. In clinical studies, the serum level of ICAM-1 in patients with atherosclerosis is higher than that in the healthy control, which decreased gradually when patients getting better. Therefore, adhesion molecules have been regarded as a key factor in the pathogenesis of atherosclerosis. Oxidized low density lipoprotein (ox-LDL) plays a critical role in the development of atherosclerosis [19]. Recent studies show that ox-LDL may be the main factor inducing endothelial cells and smooth muscle cells injury [20]. Lectin-like oxidized low-density lipoprotein (oxLDL) receptor-1 (LOX-1) is the primary endothelial receptor for oxLDL, and both its expression and function are associated with vascular inflammation. And ox-LDL has been shown to upregulate expression of LOX-1 [19]. Meanehile LOX-1 can upregulate the expressions of VCAM-1, ICAM-1, E-selectin and MCP-1 and can lead to the aggregation of monocytes to endothelial cells [21]. In this study, we observed that TNF- $\alpha$ and ICAM- 1 levels increased significantly after high-fat diet for 18 weeks, which indicating obvious vascular inflammatory reactions. Furthermore, the elevated LOX-1 level in sera indicated that there were obvious oxidative modifications of LDL in rats, which may in turn lead to further development of inflammatory in artery.

Phosphatidylinositol-3-kinase/protein kinase B (PI3K/ Akt) signaling pathway is an important cell signaling pathways in vivo, which plays an important role in the cell mobilization, migration, differentiation and apoptosis [7]. Meanwhile, PI3K/Akt signaling pathway, as one of the insulin signaling pathways, plays an important role in the glucose transport, glycogen synthesis, glycolysis and gluconeogenesis regulation, and the protein synthesis and lipolysis process [22]. Akt is the most important signaling molecule of the downstream in PI3K. Activated Akt has a wide range of biological effects including anti-apoptosis and pro survival functions by promoting the glycogen synthase kinase- $3 \beta$ (GSK-3 $\beta$ ) phosphorylation, a downstream effector of Akt [8]. In our study, decreased phosphorylation levels of Akt and GSK- $3 \beta$ in were observed in rat aorta after 18 weeks' high-fat diet, which indicated that elevated blood lipids might inhibit the activity of PI3K/Akt obviously.

Collectively, our study confirmed that short-term highfat diet can damage the function of endothelium, and the main mechanism may be the inflammatory response induced by oxidative stress, meanwhile PI3K/Akt/GSK-3 $\beta$ pathway plays an important role in the process.

\section{ACKNOWLEDGEMENTS}

This work was supported by National Natural Science Foundation of 
China $(81173584,81102845)$

\section{REFERENCES}

[1] Malloy M.J. and Kane J.P. (2012) Hyperlipidemia and cardiovascular disease. Current Opinion in Lipidology, 23, 591-592. doi:10.1097/MOL.0b013e328359f162

[2] Ingelsson, E., Massaro, J.M., Sutherland, P., et al. (2009) Contemporary trends in dyslipidemia in the Framingham Heart Study. Archives of Internal Medicine, 169, 279286. doi:10.1001/archinternmed.2008.561

[3] Ross, R. (1999) Atherosclerosis-an inflammatory disease. The New England Journal of Medicine, 340, 115-126 doi:10.1056/NEJM199901143400207

[4] Hansson, G.K. (2005) Inflammation, atherosclerosis, and coronary artery disease. The New England Journal of Medicine, 352, 1685-1695. doi:10.1056/NEJMra043430

[5] Stocker, R. and Keaney Jr., J.F. (2004) Role of oxidative modifications in atherosclerosis. Physiological Reviews, 84, 1381-1478. doi:10.1152/physrev.00047.2003

[6] Vanhoutte, P.M. (2009) Endothelial dysfunction: The first step toward coronary arteriosclerosis. Circulation Journal, 73, 595-601. doi:10.1253/circj.CJ-08-1169

[7] Franke, T.F. (2008) PI3K/Akt: Getting it right matters. Oncogene, 27, 6473-6488. doi:10.1038/onc.2008.313

[8] Park, K.W., Yang, H.M., Youn, S.W., et al. (2003) Constitutively active glycogen synthase kinase-3beta gene transfer sustains apoptosis, inhibits proliferation of vascular smooth muscle cells, and reduces neointima formation after balloon injury in rats. Arteriosclerosis, Thrombosis, and Vascular Biology, 23, 1364-1369. doi:10.1161/01.ATV.0000081633.53390.B4

[9] Jenner, A., Ren, M., Rajendran, R., et al. (2007) Zinc supplementation inhibits lipid peroxidation and the development of atherosclerosis in rabbits fed a high cholesterol diet. Free Radical Biology \& Medicine, 42, 559566. doi:10.1016/i.freeradbiomed.2006.11.024

[10] Ma, K.L., Ruan, X.Z., Powis, S.H., et al. (2008) Inflammatory stress exacerbates lipid accumulation in hepatic cells and fatty livers of apolipoprotein e knockout mice. Hepatology, 48, 770-781. doi:10.1002/hep.22423

[11] Davis, N.E. (2005) Atherosclerosis-An inflammatory process. Journal of Insurance Medicine, 37, 72-75.

[12] Jiang, W.G. and Hiscox, S. (1997) Hepatocyte growth factor/scatter factor, a cytokine playing multiple and converse roles. Histology and Histopathology, 12, 537-555.

[13] Bonomini, F., Tengattini, S., Fabiano, A., et al. (2008)
Atherosclerosis and oxidative stress. Histology and Histopathology, 23, 381-390.

[14] Del Rio, D., Stewart, A.J. and Pellegrini, N. (2005) A review of studies on malondialdehyde as toxic molecule and biological marker of oxidative stress. Nutrition, $\mathrm{Me}$ tabolism \& Cardiovascular Diseases, 15, 316-328. doi:10.1016/j.numecd.2005.05.003

[15] Luo, T. and Xia, Z. (2006) A small dose of hydrogen peroxide enhances tumor necrosis factoralpha toxicity in inducing human vascular endothelial cell apoptosis: Reversal with propofol. Anesthesia \& Analgesia, 103, 110116. doi:10.1213/01.ane.0000221183.02244.80

[16] Kleinbongard, P., Heusch, G. and Schulz, R. (2010) TNFalpha in atherosclerosis, myocardial ischemia/reperfusion and heart failure. Pharmacology \& Therapeutics, 127, 295-314. doi:10.1016/j.pharmthera.2010.05.002

[17] Frank, P.G. and Lisanti, M.P. (2008) ICAM-1: Role in inflammation and in the regulation of vascular permeability. American Journal of Physiology-Heart and Circulatory Physiology, 295, H926-H927. doi:10.1152/ajpheart.00779.2008

[18] Bourdillon, M.C., Poston, R.N., Covacho, C., et al. (2000) ICAM-1 deficiency reduces atherosclerotic lesions in double-knockout mice (ApoE( $(\ulcorner) / \mathrm{ICAM}-1(\ulcorner))$ fed a fat or a chow diet. Arteriosclerosis, Thrombosis, and Vascular Biology, 20, 2630-2635. doi:10.1161/01.ATV.20.12.2630

[19] Mitra, S., Goyal, T. and Mehta, J.L. (2011) Oxidized LDL, LOX-1 and atherosclerosis. Cardiovascular Drugs and Therapy, 25, 419-429. doi:10.1007/s10557-011-6341-5

[20] Girona, J., Manzanares, J.M. and Marimón, F., et al. (2008) Oxidized to non-oxidized lipoprotein ratios are associated with arteriosclerosis and the metabolic syndrome in diabetic patients. Nutrition, Metabolism \& Cardiovascular Diseases, 18, 380-387. doi:10.1016/j.numecd.2007.04.002

[21] Chen, M., Masaki, T. and Sawamura, T. (2002) LOX-1, the receptor for oxidized low-density lipoprotein identified from endothelial cells: Implications in endothelial dysfunction and atherosclerosis. Pharmacology \& Therapeutics, 95, 89-100. doi:10.1016/S0163-7258(02)00236-X

[22] Riley, J.K., Carayannopoulos, M.O., Wyman, A.H., et al. (2006) Phosphatidylinositol 3-kinase activity is critical for glucose metabolism and embryo survival in murine blastocysts. The Journal of Biological Chemistry, 281, 6010-6019. doi:10.1074/jbc.M506982200. 